\title{
Finding Structural Information about RF Power Amplifiers using an Orthogonal Non-Parametric Kernel Smoothing Estimator
}

\author{
Efrain Zenteno, Student Member, IEEE, Zain Ahmed Khan, Student Member, IEEE, Magnus Isaksson, Senior \\ Member, IEEE, and Peter Händel, Senior Member, IEEE
}

\begin{abstract}
A non-parametric technique for modeling the behavior of power amplifiers is presented. The proposed technique relies on the principles of density estimation using the kernel method and is suited for use in power amplifier modeling. The proposed methodology transforms the input domain into an orthogonal memory domain. In this domain, non-parametric static functions are discovered using the kernel estimator. These orthogonal, non-parametric functions can be fitted with any desired mathematical structure, thus facilitating its implementation. Furthermore, due to the orthogonality, the non-parametric functions can be analyzed and discarded individually, which simplifies pruning basis functions and provides a tradeoff between complexity and performance. The results show that the methodology can be employed to model power amplifiers, therein yielding error performance similar to state-of-the-art parametric models. Furthermore, a parameter-efficient model structure with 6 coefficients was derived for a Doherty power amplifier, therein significantly reducing the deployment's computational complexity. Finally, the methodology can also be well exploited in digital linearization techniques.
\end{abstract}

Index Terms-Power amplifier, non-parametric model, kernel, basis functions, power amplifier linearization, Digital predistortion.

\section{INTRODUCTION}

$\mathbf{E}$ NERGY-EFFICIENT power amplifiers (PAs) in wireless networks usually behave in a nonlinear fashion, thereby producing significant nonlinear distortions that degrade network performance. This creates the need for suitable behavioral models for PAs that provide simpler descriptions of nonlinear distortion mechanisms and tools for the mitigation of these effects such as digital pre-distortion (DPD) techniques [1].

Historically, behavioral models for PAs have been derived using the Volterra series [2]. The disadvantage of the Volterra series is that it involves a large number of parameters, which hinders its practical implementation. Pruning Volterra series

Copyright (c)2015 IEEE. Personal use of this material is permitted. However, permission to use this material for any other purposes must be obtained from the IEEE by sending a request to pubs-permissions@iee.org.

E. Zenteno and Z.A Khan are with the Department of Electronics, Mathematics, and Natural Sciences, the University of Gävle, SE 80176 Gävle, Sweden, and also with the Department of Signal Processing, Royal Institute of Technology KTH, SE 10044 Stockholm, Sweden (e-mail: efnzeo@hig.se, zanahn@hig.se).

M. Isaksson is with the Department of Electronics, Mathematics, and Natural Sciences, the University of Gävle, SE 80176 Gävle, Sweden (e-mail: min@hig.se).

P. Händel is with the Department of Signal Processing, Royal Institute of Technology KTH, SE 10044 Stockholm, Sweden. (e-mail: ph@kth.se) has been actively studied to provide low-complexity and high-performance behavioral models to mitigate PA nonlinear distortions [3], [4], [5]. Although pruning Volterra series has produced useful empirical models, these pruned models are general structures for smaller classes of nonlinear systems. This requires engineers to test different pruned model structures and further select the nonlinearity order and memory depths to meet certain performance requirements with a level of complexity that depends on application constraints. Hence, for a specific PA, trimming the pruned Volterra models may produce even lower complexity with the desired error performance [3], [5]. This raises the question as to whether there may exist techniques to obtain structural knowledge of a specific PA that in turn can be used to construct simpler model structures with the required model error performance. This paper presents a technique of this class.

Trimmed model structures of reduced complexity can also be obtained using sparse estimation techniques [6], [7]. However, sparse estimation techniques are usually computationally demanding and require the choosing of an initial model to be reduced. On the one hand, a general model is desired as an initial set that preserves the modeling properties. However, this involves a large set, which increases the complexity of the technique. On the other hand, starting from a small class of model structures and reducing complexity produces results that are dependent on this initial choice.

This paper presents a non-parametric method of discovering PA structural information. Thus, it assumes no a priori model structure for the PA. The proposed method considers static and dynamic distortion effects and provides a tool for analyzing the PA transfer function. In particular, the tool can be used to tailor parametric models of simpler forms. Thus, the method effectively reduces the computational complexity of the model. In PA modeling, other non-parametric techniques use statistical functions such as cumulative distribution functions (CDFs) [8], [9] and higher order statistics [10]. However, [8], [9], and [10] consider solely memoryless distortion effects, and hence, they are ineffective at characterizing and compensating PA distortion caused by memory effects.

The proposed technique is based on non-parametric density estimation [11] referred to as the kernel smoothing estimator method or simply the kernel method [12]. Compared to polynomial-based PA models, the kernel method can estimate nonlinear functions of high nonlinearity order without numerical difficulties [13]. Furthermore, the kernel method uses 
window averages, which are less computationally demanding than the matrix (pseudo) inversions required in the least square methods. Finally, the kernel estimator has strong statistical properties: asymptotic convergence [14], optimal estimation in the square error sense given a limited number of samples and robustness against noise sources [15]. All these properties make the kernel method a suitable candidate for modeling PAs.

The work reported in this paper reviews the modeling methodology presented in [16] and performs the adaptations necessary for the PA measurement scenario. PAs are characterized by band-limited, complex baseband equivalent signals, which make the method in [16] unsuitable for PA modeling. However, with the adaptations proposed in [17] and our previous study [13], we obtain a methodology and method suitable for this application. In contrast to traditional PA modeling techniques, the work reported here transforms the input sample domain into an orthogonal domain, where the model structure is obtained using the kernel method. The orthogonal domain simplifies the analysis of the PA transfer function; allowing the addition or removal of basis functions provides a tradeoff between complexity and performance. This result can be transferred to the original sample domain, thereby reversing the orthogonalization process (linear combination) and obtaining model structures that are comparable in performance with the state-of-the-art methods but with reduced computational requirements for deployment.

\section{PA MODELING}

\section{A. PA model}

Let $u(n)$ and $y(n)$ denote the $n$-th complex-valued sample of the baseband signals corresponding to the input and output of a PA, respectively. The PA nonlinear transfer function is approximated by [16]

$$
\begin{aligned}
y(n) & =\sum_{m_{1}} f_{m_{1}}\left(u\left(n-m_{1}\right)\right)+ \\
& +\sum_{m_{1}} \sum_{m_{2}} f_{m_{1}, m_{2}}\left(u\left(n-m_{1}\right), u\left(n-m_{2}\right)\right)+\ldots \\
& +\sum_{m_{1}} \ldots \sum_{m_{p}} f_{m_{1}, m_{2}, \ldots, m_{p}}\left(u\left(n-m_{1}\right), \ldots, u\left(n-m_{p}\right)\right),
\end{aligned}
$$

where $f_{m_{1}}(\cdot), f_{m_{1}, m_{2}}(\cdot, \cdot)$ and $f_{m_{1}, m_{2}, \ldots, m_{p}}(\cdot, \ldots, \cdot)$ are nonlinear static functions whose domain dimensions are 1,2 and $p$, respectively. The summations go up to $M$ subject to $0 \geq m_{1}>m_{2}>\ldots>m_{p} \geq M$, where $M$ is the maximum memory depth considered.

The Volterra series is a special form of (1), which can be obtained when $f_{m_{1}}(\cdot), f_{m_{1}, m_{2}}(\cdot, \cdot)$ and $f_{m_{1}, m_{2}, \ldots, m_{p}}(\cdot, \ldots, \cdot)$ are defined as the scaled product of their arguments. The static functions in (1) can represent high nonlinearity orders of the Volterra series. In particular, high nonlinearity orders are coupled to different memory depths, which is the cause of the rapid growth in the number of parameters in the Volterra series. Despite the different features of (1) compared to the Volterra series, both suffer from high dimensionality. Considering $0 \geq m_{1}>m_{2}>\ldots>m_{p} \geq M$, the system in (1) has a total number of additive functions of

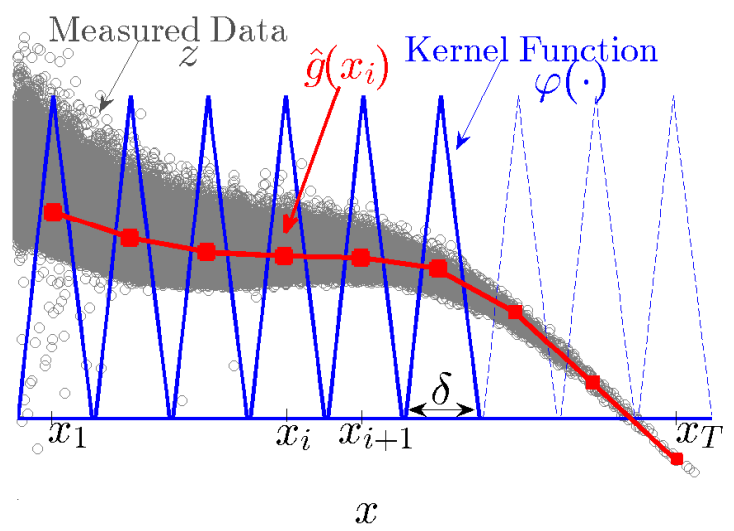

Fig. 1. Illustration of the estimation of the function $g(\cdot)$ at fixed grid $x_{i}$ through a triangular kernel $\varphi(\cdot)$. The value $\hat{g}\left(x_{i}\right)$ is obtained as the weighted average of the output data $z$ through the kernel $\varphi(\cdot)$.

$\sum_{d=1}^{p}\left(\begin{array}{c}M+1 \\ d\end{array}\right)=\sum_{d=1}^{p} \frac{(M+1) !}{d !(M+1-d) !}$, with $p$ being the highest dimension of the functions in (1) and ! denoting the factorial operator. The high dimensionality increases the computational complexity of the identification and deployment of the models. Thus, we analyze the relationship in (1) and study possible simplifications to allow it to be suitable for PA modeling.

\section{B. Kernel method brief}

The kernel estimator as applied to the estimation of a static nonlinear input output relation [12] is briefly reviewed. Consider the set of real-valued input data $\{x(n)\}_{n=0}^{N-1}$ passed through an unknown, static nonlinear function $g(\cdot)$ and producing the output $\{z(n)\}_{n=0}^{N-1}$, that is, $z(n)=g(x(n))$ for $n=0, \ldots, N-1$. Then, the static function $g(\cdot)$ can be estimated at a scalar point $x_{i}$ as illustrated in Fig. 1 by the kernel (window) average [12]

$$
\hat{g}\left(x_{i}\right)=\sum_{n=0}^{N-1} \frac{\varphi\left(\frac{x(n)-x_{i}}{\delta}\right)}{\sum_{\ell=0}^{N-1} \varphi\left(\frac{x(\ell)-x_{i}}{\delta}\right)} z(n),
$$

where the grid of points $x_{i}$ for $i=1, \ldots, T$ span the amplitude support of $x(n)$ and $\varphi(\cdot)$ is the kernel with aperture $\delta$. Here, the triangular kernel is preferred because it is the minimum mean square error estimator for a limited number of samples and is robust against noise [15], that is,

$$
\varphi(x)= \begin{cases}1-|x| & \text { if }|x| \leq 1 \\ 0 & \text { if }|x|>1\end{cases}
$$

with $|\cdot|$ denoting the absolute value. Equation (2) is evaluated only if the denominator is different from zero. In this paper, a linear interpolation between the two nearest neighbors is employed to compute $\hat{g}(\cdot)$ for an arbitrary input within the amplitude support of $x(n)$.

The kernel method is not directly suited to simulating PA behavior. First, PA measurements show a significant correlation between different samples of the input signal $u(n)$. The sample correlation makes the output of the functions 
$f_{m_{1}}(\cdot), f_{m_{1}, m_{2}}(\cdot, \cdot)$ and $f_{m_{1}, m_{2}, \ldots, m_{p}}(\cdot, \ldots, \cdot)$ jointly correlated. Thus, their estimation needs to simultaneously account for all of them.

Second, the kernel method is intended for real-valued data. For complex-valued data widely available within PA instrumentation, the static functions in (1) are functions of complexvalued inputs and outputs [13]. This results in the method having large computational and storage requirements. In the following, a method that addresses these drawbacks is outlined and discussed with simplifications (complexity reductions) of (1) suitable for PA modeling.

\section{Removing correlation by orthogonalization}

The PA input signal is band limited and digitized with oversampling, yielding $u(n)$. As a result of the oversampling, this discrete signal has a significant correlation between different samples. However, removing the correlation in $u(n)$ can be viewed as orthogonalizing it [18], which can be efficiently performed using the Gram-Schmidt (GS) process.

According to (1), the signal set to be orthogonalized lies in the space

$$
\mathcal{U}=\{u(n), \ldots, u(n-M)\}
$$

The GS process yields an orthogonal set $\overline{\mathcal{U}}=$ $\{\bar{u}(n), \ldots, \bar{u}(n-M)\}$, where $\bar{u}(n)=u(n)$, followed by an iterative process for $k=1, \ldots, M$,

$$
\bar{u}(n-k)=u(n-k)-\sum_{\ell=0}^{k-1} P_{k, \ell} \bar{u}(n-\ell) .
$$

The scalar $P_{k, \ell}$ is a projection of the signal $\bar{u}(n-\ell)$ over $u(n-k)$ defined by

$$
P_{k, \ell}=\sum_{n} u^{*}(n-k) \bar{u}(n-\ell),
$$

where $*$ denotes the complex conjugate operator. Note that the GS process involves a linear combination, and thus, it can be reversed without any loss of information. Assuming that $u(n)$ is a wide-sense stationary stochastic process with $k$-th auto-correlation lag denoted by $r_{u}(k)$, we note that the projections can be a priori calculated using $r_{u}(k)$. This leads to a computationally preferable method; e.g., a rectangularshaped power spectral density of a Long-Term Evolution (LTE) signal yields a sinc-shaped auto-correlation function.

\section{Real-valued PA input signal}

The distortion produced by PAs operating within wireless networks can be regarded as amplitude dependent [4], [13]. Thus, considering solely the amplitude of the signals in the orthogonal input set $\overline{\mathcal{U}}$ yields the set

$$
|\overline{\mathcal{U}}|=\{|\bar{u}(n)|, \ldots,|\bar{u}(n-M)|\},
$$

which will be the input to our kernel estimator, e.g., $x(n)=$ $|\bar{u}(n)|$. To compensate for the phase contribution, the output signal $y(n)$ is transformed as

$$
z(n)=y(n) e^{-j \angle \bar{u}(n)} .
$$

By applying the GS process to the input set $\mathcal{U}$ followed by the real-value transformation, the system (1) becomes

$$
\begin{aligned}
z(n) & =\sum_{m_{1}} g_{m_{1}}\left(x\left(n-m_{1}\right)\right)+ \\
& +\sum_{m_{1}} \sum_{m_{2}} g_{m_{1}, m_{2}}\left(x\left(n-m_{1}\right), x\left(n-m_{2}\right)\right)+\ldots \\
& +\sum_{m_{1}} \ldots \sum_{m_{p}} g_{m_{1}, m_{2} \ldots m_{p}}\left(x\left(n-m_{1}\right), \ldots, x\left(n-m_{p}\right)\right),
\end{aligned}
$$

with complex-valued $\quad g_{m_{1}}(\cdot), \quad g_{m_{1}, m_{2}}(\cdot, \cdot) \quad$ and $g_{m_{1}, m_{2}, \ldots, m_{p}}(\cdot, \ldots, \cdot)$ as the orthogonal counterparts of the functions in (1) but with real-valued arguments. This reduces the estimation dimension required in the kernel method. The system (9) has similar features as (1) for modeling nonlinear behavior. However, in contrast to (1), it has orthogonal basis functions. Thus, their estimation can be performed individually, and each basis contribution can be separately analyzed.

\section{E. Complexity reduction of (1)}

Despite using real-valued input signals, the complexity of the estimation of a multi-variable function in (9) remains high; e.g., a $p$-th variable function estimated at $T$ points for each variable gives a total of $T^{p}$ estimation points. Thus, the memory requirements and data manipulation increase exponentially with $p$, leading to the well-known curse of dimensionality problem. In an attempt to alleviate this, a $p$-variable function $g_{m_{1}, \ldots, m_{p}}(\cdot, \ldots, \cdot)$ is approximated as a sum of single-variable functions:

$$
\begin{aligned}
& g_{m_{1}, \ldots, m_{p}}\left(x\left(n-m_{1}\right), \ldots, x\left(n-m_{p}\right)\right) \\
& \sum_{k=1}^{p} h_{m_{k}}\left(x\left(n-m_{k}\right) \prod_{\substack{d=1 \\
d \neq k}}^{p} x\left(n-m_{d}\right)\right) .
\end{aligned}
$$

Thus, the single-variable functions $h_{m_{k}}(\cdot)$ can be estimated using (2). In PA modeling, (10) has been motivated from a physical [19] and signal processing perspective [17]. Note that the new single-variable $x\left(n-m_{k}\right) \prod_{\substack{d=1 \\ d \neq k}}^{p} x\left(n-m_{d}\right)$ can similarly be considered in the non-orthogonal domain $\mathcal{U}$ by augmenting it as

$$
\mathcal{U}^{\prime}=\left[\mathcal{U},\left\{u\left(n-m_{k}\right) \prod_{\substack{d=1 \\ d \neq k}}^{p}\left|u\left(n-m_{d}\right)\right|\right\}_{m_{k}=0}^{M}\right]
$$

for $p=2, \ldots, M$. The orthogonalization of the data set $\mathcal{U}^{\prime}$ is performed using the GS procedure.

\section{F. Summary and implementation}

Consider the data set of complex-valued input and output measurements $\{u(n)\}$ and $\{y(n)\}$, respectively. The nonparametric modeling approach begins by creating the input space $\mathcal{U}^{\prime}$ as indicated by (11) for the chosen maximum memory depth $M$. The implementation of the method can proceed by storing $\mathcal{U}^{\prime}$ as a matrix whose columns are the basis in (11). This matrix is column-wise orthogonalized using the 
GS process, therein yielding an orthogonal matrix. Only magnitude entries of the orthogonal matrix are retained according to (7), rendering a magnitude matrix. Finally, each column of the magnitude matrix is used as a domain to estimate single-variable functions with the kernel method in (2). The results show that significant contribution to the model output continues to originate from a few single-variable functions. Thus, due to the orthogonality, the non-contributing functions can be eliminated from the model structure (remove the corresponding columns) while retaining the obtained model performance.

\section{EXPERIMENTAL}

\section{A. Measurement setup}

The measurement setup includes a vector signal generator R\&S SMU 200A that is used to excite the PA. The PA output is measured using a wideband down converter and a high-performance analog-to-digital converter (ADC) with 14-bit resolution operated with a $400 \mathrm{MHz}$ sampling rate. The amplifier being tested is the MRF8S21120HS Doherty amplifier with $14 \mathrm{~dB}$ linear gain, an operation frequency in the 2.1-2.2 $\mathrm{GHz}$ band, and a rating of $46 \mathrm{dBm}$ output power operated at approximately $3 \mathrm{~dB}$ of compression.

Two independent excitation signals with bandwidths of 12 and $24 \mathrm{MHz}$ are generated. These excitations are noise-like signals with peak-to-average power ratios of 11.2 and 11.4 $\mathrm{dB}$, respectively. The excitations were created in a $\mathrm{PC}$ using $10^{5}$ complex-valued samples uploaded to the generator and upconverted to $2.14 \mathrm{GHz}$ to excite the PA. The measurements consist of $10^{5}$ complex-valued samples for the input and the output of the amplifier with post-processing time and phase delay compensation [1]. The non-parametric structure is obtained using $10 \%$ (estimation phase) of the measured data, and the remaining $90 \%$ (validation phase) is used to evaluate the modeling error.

\section{B. Results}

1) User-defined parameters: In the proposed method, the number of grid points $T$ and the kernel aperture $\delta$ are userdefined parameters. Fig. 2 shows the normalized mean square error (NMSE) contours over both $\delta$ and $T$ in a linearly spaced grid. The kernel aperture $\delta$ is shown as a percentage of the span of the input signal.

The number of grid points $T$ sets the resolution of the static function and the kernel aperture $\delta$ sets the size of the input neighborhood to perform the average (estimation) (cf. Fig. 1). Thus, a large value of $T$ and a small value of $\delta$ are desired to produce an accurate estimation. However, for a fixed number $N$ of measurement samples, decreasing $\delta$ may degrade the performance because the number of measurement samples in each kernel function decreases, and hence, its average (estimate) has increased variance (less reliability). This is the reason for the loss in performance for small values of $\delta$ in Fig. 2. Because $T$ is the number of entries to be stored, $T$ can be chosen based on the available memory resources, and as a rule of thumb, the kernel aperture can be set as $\delta=1 / T$ to avoid the performance degrading effects, cf. Fig. 2. The choice

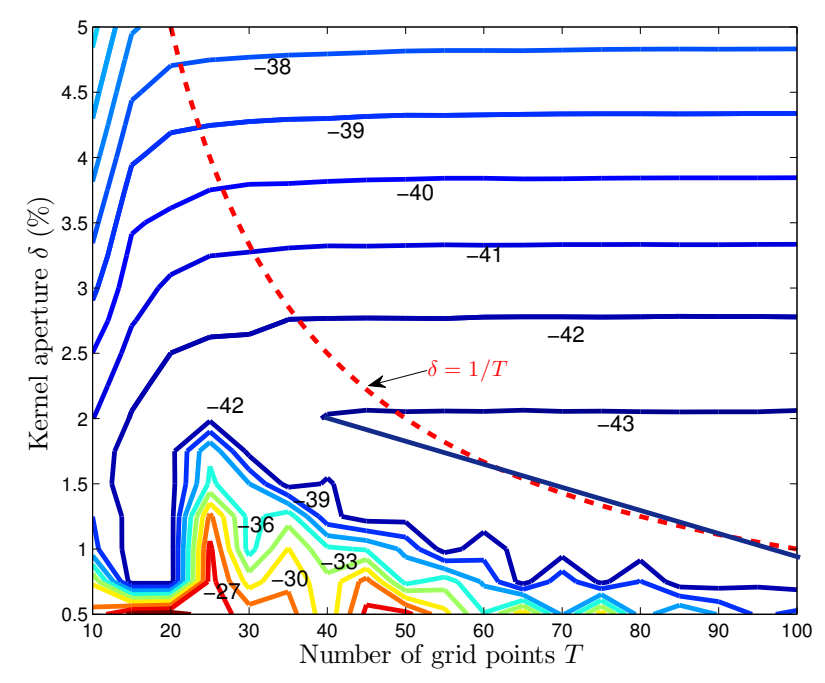

Fig. 2. NMSE (in $\mathrm{dB}$ ) as a function of the number of grid points $T$ and the kernel aperture $\delta$ (as a percentage of the span of the input signal).

TABLE I

INDIVIDUAL CONTRIBUTIONS OF THE FUNCTIONS FOR TWO DIFFERENT SIGNAL BANDWIDTHS

\begin{tabular}{c|c|c|c|c}
\hline \hline \multirow{2}{*}{ Basis } & \multicolumn{2}{|c|}{$12 \mathrm{MHz}$} & \multicolumn{2}{c}{$24 \mathrm{MHz}$} \\
\cline { 2 - 5 } & NMSE & ACEPR & NMSE & ACEPR \\
{$[\mathrm{dB}]$} & {$[\mathrm{dB}]$} & {$[\mathrm{dB}]$} & {$[\mathrm{dB}]$} \\
\hline$\hat{g}_{0}(\cdot)$ & $\mathbf{- 3 8 . 3}$ & -54.9 & $\mathbf{- 3 2 . 5}$ & -50.8 \\
$\hat{g}_{1}(\cdot)$ & $\mathbf{- 5 . 0}$ & 0.0 & $\mathbf{- 6 . 7}$ & -0.4 \\
$\hat{g}_{2}(\cdot)$ & -0.3 & 0.0 & $\mathbf{- 2 . 3}$ & 0.3 \\
$\hat{g}_{3}(\cdot)$ & -0.0 & 0.0 & -0.4 & 0.0 \\
\hline$\hat{g}_{0,1}(\cdot)$ & -0.1 & -0.3 & $\mathbf{- 0 . 5}$ & -0.2 \\
$\hat{g}_{0,2}(\cdot)$ & -0.1 & 0.0 & -0.1 & 0.1 \\
$\hat{g}_{0,3}(\cdot)$ & -0.0 & 0.0 & -0.0 & 0.0 \\
$\hat{g}_{1,2}(\cdot)$ & -0.0 & 0.0 & 0.0 & 0.0 \\
$\hat{g}_{1,3}(\cdot)$ & 0.0 & 0.0 & 0.0 & 0.0 \\
$\hat{g}_{2,3}(\cdot)$ & 0.0 & 0.0 & 0.0 & 0.0 \\
\hline$\hat{g}_{0,1,2}(\cdot)$ & -0.1 & 0.0 & -0.1 & -0.0 \\
$\hat{g}_{0,1,3}(\cdot)$ & 0.0 & 0.0 & 0.0 & 0.0 \\
$\hat{g}_{0,2,3}(\cdot)$ & 0.0 & 0.0 & 0.0 & 0.0 \\
$\hat{g}_{1,2,3}(\cdot)$ & 0.0 & 0.0 & 0.0 & 0.0 \\
\hline Total & -43.9 & -55.2 & -42.6 & -51.0 \\
\hline \hline
\end{tabular}

$\delta=1 / T$ has the advantage of efficiently using all training data for estimating the non-parametric model.

2) Modeling performance: Using $T=70$ and $\delta=1 / 70$, a non-parametric model of the PA is obtained for the two input signals under consideration. Table I shows the individual contributions of the basis functions to the NMSE and to the adjacent channel error power ratio (ACEPR) [1] for these two signals. As observed in Table I, the function $\hat{g}_{0}(x)$ contributes -38.3 and $-32.5 \mathrm{~dB}$ of the total NMSE for the 12 and 24 $\mathrm{MHz}$ signals, respectively. This function is the largest model contributor because it captures linear and nonlinear static effects.

For the $12 \mathrm{MHz}$ signal, the NMSE is dominated by the contributions of $\hat{g}_{0}(x)$ and $\hat{g}_{1}(x)$, which provide a combined 

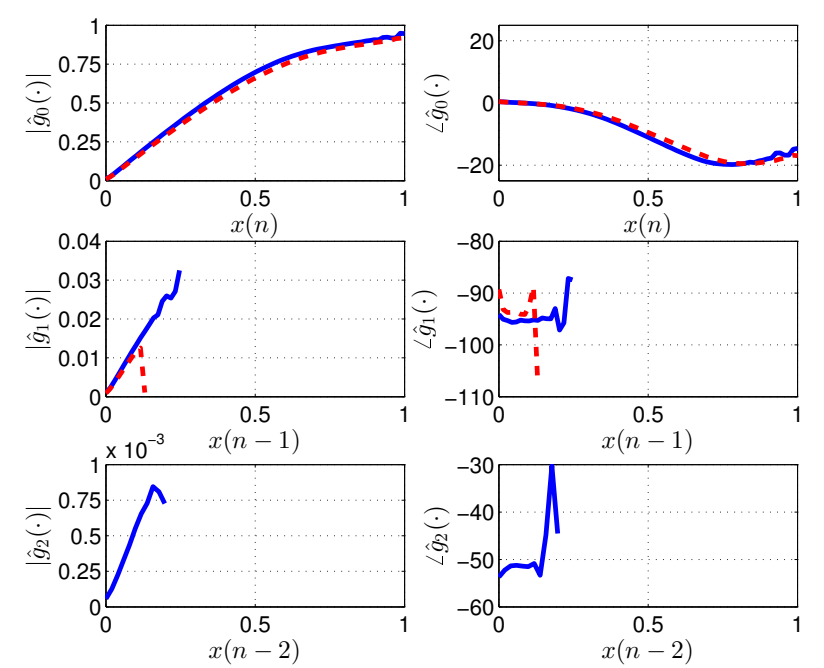

Fig. 3. Amplitude and phase (in degrees) of the estimated single-variable functions $\hat{g}_{0}(\cdot), \hat{g}_{1}(\cdot)$, and $\hat{g}_{2}(\cdot)$ for the $24 \mathrm{MHz}$ signal (solid - blue) and $\hat{g}_{0}(\cdot)$ and $\hat{g}_{1}(\cdot)$ for the $12 \mathrm{MHz}$ (dashed - red) signal. The function $\hat{g}_{2}(\cdot)$ is not presented for the $12 \mathrm{MHz}$ signal because its contribution is negligible.

NMSE of $-43.3 \mathrm{~dB}$. However, in the $24 \mathrm{MHz}$ input signal, the contribution to the NMSE from the function $\hat{g}_{2}(x)$ increases from -0.3 to $-2.3 \mathrm{~dB}$, thereby revealing the impact of memory effects caused by the increase in signal bandwidth. Note that the contributions from the 2- and 3-variable functions is negligible in the $12 \mathrm{MHz}$ case, and $\hat{g}_{0,1}(\cdot)$ significantly increases its contribution when increasing the signal bandwidth from 12 to $24 \mathrm{MHz}$ from -0.1 to $-0.5 \mathrm{~dB}$. In terms of the ACEPR, only $\hat{g}_{0}(x)$ provides a significant contribution for both signal bandwidths. The static nonlinear distortion is modeled by $\hat{g}_{0}(\cdot)$, and the linear and nonlinear dynamics are described by $\hat{g}_{1}(\cdot)$ and $\hat{g}_{2}(\cdot)$. Because these functions are arbitrary, the non-parametric structure can model memory effects coupled to strong nonlinearities, which are one of the causes of poor behavior in polynomial-based model methodologies.

The functions contributing more than $-2 \mathrm{~dB}$ to the NMSE are shown in Fig. 3 for both signal bandwidths. Despite the two signals being independently created and having different bandwidths, the estimated functions are similar to each other, which suggests that the method obtains structural information about the modeled PA.

The power spectral density (PSD) of the input, output and model error evolution are plotted in Fig. 4 for the $24 \mathrm{MHz}$ bandwidth signal. The model was updated sequentially to include the first six basis functions of Table I. The in-band error spectrum decreases with the addition of basis functions, and the out-of-band error is suppressed by the use of $\hat{g}_{0}(x)$. These two observations are in accordance with Table I.

An advantage of the proposed method is that we can utilize the estimated basis functions (cf. Fig. 3) to build a parametric model of the PA. These parametric models can be of any form; they can be chosen to ease implementation and identification or to maximize performance. We seek a parameter efficient representation using a polynomial family as an example. Thus, the function $\hat{g}_{0}(\cdot)$ is modeled with a seventh-order polynomial,

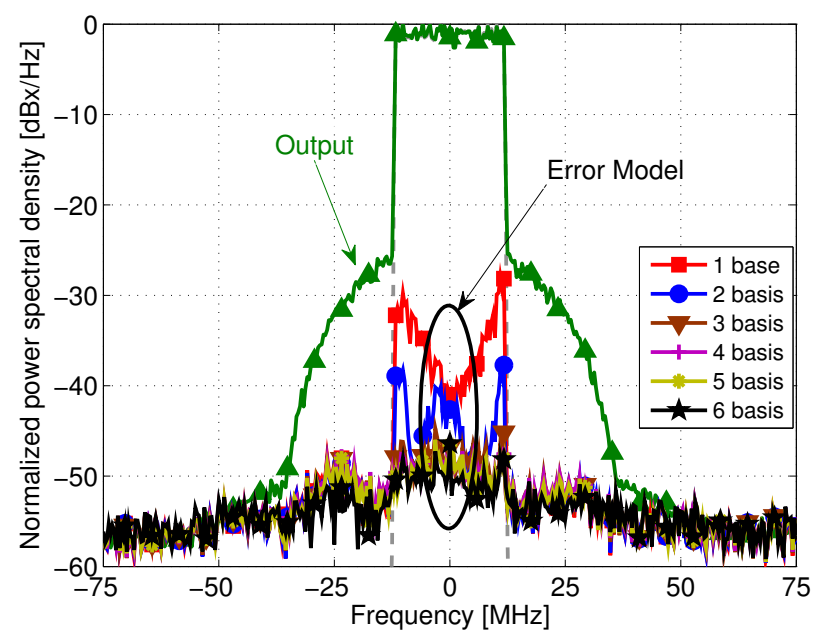

Fig. 4. Power spectral density of the output and error model obtained by sequentially including the first 6 basis functions indicated in Table I in the nonparametric model for the $24 \mathrm{MHz}$ signal.

and $\hat{g}_{1}(\cdot)$ and $\hat{g}_{2}(\cdot)$ are modeled with linear polynomials (cf. Fig. 3), that is,

$$
z(n)=\sum_{p=1}^{4} \gamma_{p} \bar{u}(n)|\bar{u}(n)|^{2(p-1)}+\gamma_{5} \bar{u}(n-1)+\gamma_{6} \bar{u}(n-2) \text {. }
$$

Due to the orthogonality, the remainder of the functions in Table I can be discarded without affecting the model performance. Furthermore, by replacing the orthogonal variables for the linear combination of their non-orthogonal counterparts given in the GS process (Section II-C), the parametric description of this model becomes

$y(n)=\sum_{p=1}^{4} \alpha_{p} u(n)|u(n)|^{2(p-1)}+\alpha_{5} u(n-1)+\alpha_{6} u(n-2)$.

This 6-parameter model $\left[\alpha_{1}, \ldots, \alpha_{6}\right]$ can be identified using linear regression techniques, which are commonly used in PA modeling [4].

Fig. 5 shows the NMSE performance versus the complexity incurred when using the feed-forward model. The complexity is measured in floating point operations (FLOPs) [20]. Fig. 5 compares the proposed method with several parametric models, such as the static nonlinear, memory polynomial, generalized memory polynomial [4], Multi-LUT [21], Volterra [2] and Kautz-Volterra [22] models, and non-parametric models, such as the Histogram model [8]. Different points correspond to different model settings (nonlinearity order and memory depth) being tested.

Although, in general, it is possible to trade reduced NMSE for increased complexity, these settings need to be chosen with care to obtain optimal performance for the level of complexity incurred, as observed by the performance dispersion in Fig. 5. Moreover, some model settings with increased model complexity degrade the NMSE performance, as observed in Fig. 5, which is due to an unsuitable model being chosen. For example, a static model of high nonlinearity order may have 


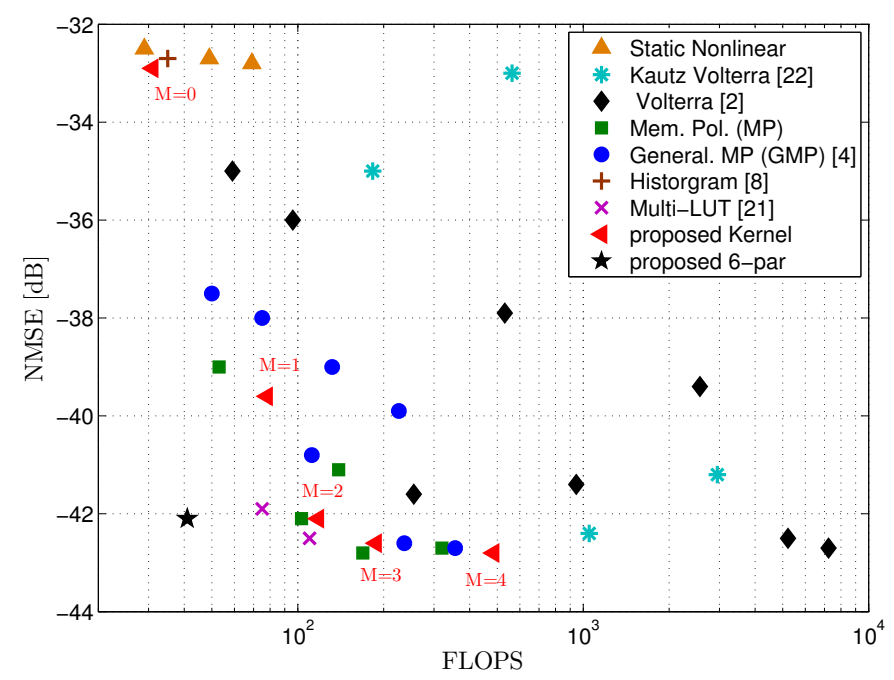

Fig. 5. NMSE of the $24 \mathrm{MHz}$ signal versus the number of FLOPS incurred when using the feed-forward model for several modeling techniques. The kernel method uses $\delta=1 / T, T=70$ with $M$ memory depth. Different NMSE values are obtained by changing the settings in the methods.

large complexity but remains unable to model dynamic effects, thereby providing limited performance. Similar arguments can be made for memory depths. Moreover, these detrimental effects have been discussed in previous studies [20]. The proposed kernel method has good performance/complexity compared to state-of-the-art parametric models. Finally, the proposed method was used to construct a parameter-efficient structure (6-parameter model), which provides the best NMSE for its reduced level of complexity because it was specifically tailored for the PA.

\section{Digital PRE-Distortion (DPD)}

The proposed method is tested as a pre-distorter compensating for nonlinear distortions at the PA output. The nonparametric structure is obtained using an inverse learning architecture, in which input and output are interchanged [23]. To increase efficiency, a clipping technique [24] has been applied to the $24 \mathrm{MHz}$ input signal, therein reducing its PAPR from 11.4 to $8.8 \mathrm{~dB}$. However, care must be exercised because clipping techniques introduce in-band and out-of-band errors.

From (9), the function $g_{0}(\cdot)$ of the DPD model has to be the inverse of the same function in the feed-forward model. For the remainder of the functions in the DPD model, they have to be the negative of their counterparts in the feed-forward model (same amplitude but with the phase shifted by 180 degrees). This is depicted in Fig. 6, where the feed-forward and inverse (DPD) estimated non-parametric functions are plotted.

The PA operates at $-25 \mathrm{~dB}$ of NMSE and $-36 \mathrm{~dB}$ of ACPR, respectively, without DPD. The pre-distorted PA with the outlined method produces an NMSE and ACPR of -42 and $-49.5 \mathrm{~dB}$, respectively, which shows its effectiveness in compensating nonlinear distortion.
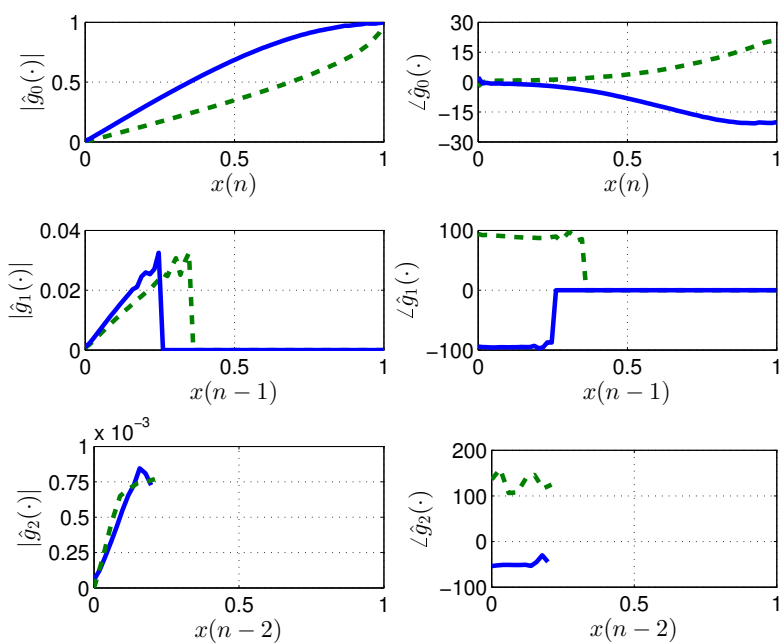

Fig. 6. Amplitude and phase (in degrees) of the estimated functions of the feed-forward model in solid blue and inverse (DPD) model in dashed green. The inverse model was estimated using the inverse learning architecture [23].

\section{CONCLusions}

A non-parametric method of modeling RF power amplifiers is presented. The method does not assume an a priori model structure of the PA. Thus, basis functions that describe its behavior are estimated during the identification process, leading to the development of tailored parametric models. These tailored models can be fitted with any desired structure, which eases its implementation. In particular, parameter-efficient models with small errors can be obtained, thereby reducing the implementation and deployment computational costs.

The presented method is based on the kernel estimator, which solely performs sample averages and hence does not suffer from numerical instabilities. Furthermore, adaptive schemes can be made using running averages, which require low computational resources and feature real-time implementations. The proposed methodology can lead to the low computational resource implementation of look-up tables (LUTs) for adaptive digital pre-distortion (linearization).

\section{REFERENCES}

[1] M. Isaksson, D. Wisell, and D. Rönnow, "A comparative analysis of behavioral models for RF power amplifiers," IEEE Trans. Microw. Theory Tech., vol. 54, no. 1, pp. 348 -359, Jan. 2006.

[2] M. Schetzen, The Volterra and Wiener Theories of Nonlinear Systems. New York: Wiley \& Sons, 1980.

[3] Y.-J. Liu, J. Zhou, W. Chen, and B.-H. Zhou, "A robust augmented complexity-reduced generalized memory polynomial for wideband RF power amplifiers," IEEE Trans. Ind. Electron., vol. 61, no. 5, pp. 23892401, May. 2014.

[4] D. Morgan, Z. Ma, J. Kim, M. Zierdt, and J. Pastalan, "A generalized memory polynomial model for digital predistortion of RF power amplifiers," IEEE Trans. Signal Process., vol. 54, no. 10, pp. $3852-3860$, Oct. 2006.

[5] $\mathrm{X}$. Yu and H. Jiang, "Digital predistortion using adaptive basis functions," IEEE Trans. Circuits Syst. I, Reg. Papers, vol. 60, no. 12, pp. 3317-3327, Dec 2013.

[6] J. Reina-Tosina, M. Allegue-Martinez, C. Crespo-Cadenas, C. Yu, and S. Cruces, "Behavioral modeling and predistortion of power amplifiers under sparsity hypothesis," IEEE Trans. Microw. Theory Tech., vol. 63, no. 2, pp. 745-753, Feb 2015. 
[7] E. Zenteno, S. Amin, M. Isaksson, P. Händel, and D. Rönnow, "Combating the dimensionality of nonlinear MIMO amplifier predistortion by basis pursuit," European Microwave Conf. (EuMC), pp. 833-836, Oct. 2014.

[8] D. Huang, X. Huang, and H. Leung, "Nonlinear compensation of high power amplifier distortion for communication using a histogram-based method," IEEE Trans. Signal Process., vol. 54, no. 11, pp. 4343-4351, Nov. 2006.

[9] Z. Zhu, X. Huang, and M. Caron, "Theoretical and experimental studies of a probabilistic-based memoryless PA linearization technique," Circuits, Systems, and Signal Processing, vol. 32, no. 6, pp. 3031-3057, 2013.

[10] Z. Zhu, X. Huang, M. Caron, and H. Leung, "A blind AM/PM estimation method for power amplifier linearization," IEEE Signal Process. Lett., vol. 20 , no. 11 , pp. $1042-1045$, Nov. 2013.

[11] M. Rosenblatt, "Remarks on some nonparametric estimates of a density function," Ann. of Math. Stat., vol. 27, no. 3, pp. 832-837, 1956.

[12] A. A. Georgiev, "Nonparametric system identification by kernel methods," IEEE Trans. Autom. Control, vol. 29, no. 4, pp. 356-358, Apr 1984.

[13] Z. Khan, E. Zenteno, M. Isaksson, and P. Händel, "Density estimation models for strong nonlinearities in RF power amplifiers," in Asia Pacific Microw. Conf., Sendai, APMC., Dec 2014, pp. -.

[14] V. Epanechnikov, "Non-parametric estimation of a multivariate probability density," Theory of Probability \& Its Applications, vol. 14, no. 1, pp. 153-158, 1969.

[15] E.-W. Bai and Y. Liu, "Recursive direct weight optimization in nonlinear system identification: A minimal probability approach," IEEE Trans. Autom. Control, vol. 52, no. 7, pp. 1218-1231, Jul. 2007.

[16] E.-W. Bai, "Non-parametric nonlinear system identification: A datadriven orthogonal basis function approach," IEEE Trans. Autom. Control, vol. 53 , no. 11 , pp. 2615-2626, Dec. 2008.

[17] H. Jiang and P. A. Wilford, "Digital predistortion for power amplifiers using separable functions," IEEE Trans. Signal Process., vol. 58, no. 8, pp. 4121-4130, Aug 2010.

[18] S. J. Leon and W. Gander, "Gram-schmidt orthogonalization: 100 years and more," Numerical Linear Algebra with Applications, vol. 20, no. 3, pp. 492 - 532, May 2013.

[19] T. Cunha, E. Lima, and J. Pedro, "Validation and physical interpretation of the power-amplifier polar volterra model," IEEE Trans. Microw. Theory Tech., vol. 58, no. 12, pp. 4012-4021, Dec. 2010.

[20] A. Tehrani, H. Cao, S. Afsardoost, T. Eriksson, M. Isaksson, and C. Fager, "A comparative analysis of the complexity/accuracy tradeoff in power amplifier behavioral models," IEEE Trans. Microw. Theory Tech., vol. 58, no. 6, pp. 1510-1520, Jun. 2010.

[21] P. Gilabert, A. Cesari, G. Montoro, E. Bertran, and J.-M. Dilhac, "Multilookup table fpga implementation of an adaptive digital predistorter for linearizing RF power amplifiers with memory effects," IEEE Trans. Microw. Theory Tech., vol. 56, no. 2, pp. 372-384, Feb 2008.

[22] M. Isaksson and D. Rönnow, "A parameter-reduced Volterra model for dynamic RF power amplifier modeling based on orthonormal basis functions," Int. J. RF and Microw. Comput.-Aided Eng., vol. 17, no. 6, pp. 542-551, 2007.

[23] C. Eun and E. Powers, "A new Volterra predistorter based on the indirect learning architecture," IEEE Trans. Signal Process., vol. 45, no. 1, pp. 223-227, 1997

[24] T. Jiang and Y. Wu, "An overview: Peak-to-average power ratio reduction techniques for OFDM signals," IEEE Trans. Broadcast., vol. 54, no. 2, pp. 257-268, Jun. 2008.

Efrain Zenteno (S'10) received the B.S. degree from San Agustin University, Arequipa, Peru, in 2004. He is currently pursuing the Ph.D. degree at the Department of Signal Processing, Royal Institute of Technology KTH, Stockholm, Sweden. During 2005, he was a field engineer at the electric company SEAL. He received the M.Sc.degree in electronics/telecommunications engineering from the University of Gävle, Gävle, Sweden, in 2008. From 2008 to 2010, he was with the Program of Telecommunications Engineering, Universidad Catlica San Pablo, Arequipa, Peru. He is currently with the Department of Electronics, Mathematics, and Natural Sciences, University of Gävle, and with the Department of Signal Processing, Royal Institute of Technology KTH. His main interests are instrumentation, measurements, and signal processing algorithms for communications.
Zain Ahmed Khan (S'13) received his B.S. degree in electronics engineering from Ghulam Ishaq Khan Institute, Pakistan, in 2005 and his M.S. degree in communication and signal processing from Technical University Ilmenau, Germany in September 2013. Currently, he is a PhD student at the Department of Signal Processing, Royal Institute of Technology KTH, Stockholm, Sweden and is working at the Department of Electronics, Mathematics, and Natural Sciences, University of Gävle. He started his $\mathrm{PhD}$ in December 2013 and is working on the behavioral modeling and digital predistortion of RF power amplifiers with particular focus on MIMO transmitters.

Magnus Isaksson (S'98-M'07-SM'12) received the M.Sc. degree in microwave engineering from the University of Gävle, Gävle, Sweden, in 2000, the Licentiate degree from Uppsala University, Uppsala, Sweden, in 2006, and the Ph.D. degree from the Royal Institute of Technology, Stockholm, Sweden, in 2007. In 2012, he was appointed Docent in Telecommunications in the Royal Institute of Technology (KTH), Stockholm, Sweden. During 1989-1999, he was with the Televerket, Sweden, working on communication products. Since 1999, he is with the Department of Electronics, Mathematics, and Natural Sciences at the University of Gävle, Gävle, Sweden, where he is currently a professor of electronics and department head. His research activities are in signal processing algorithms for radio-frequency measurements and characterization, modeling, and compensation of nonlinear microwave devices and systems. Dr. Isaksson is the author or co-author of many published peerreview journal articles, books, and conference proceedings in the area, and is currently Head of research within the fields of mathematics, and natural sciences at the University of Gävle, Gävle, Sweden.

Peter Händel (S'88-M'94-SM'98) received the Ph.D. degree from Uppsala University, Uppsala, Sweden, in 1993. From 1987 to 1993, he was with Uppsala University. From 1993 to 1997, he was with Ericsson AB, Kista, Sweden. From 1996 to 1997 , he was a Visiting Scholar with the Tampere University of Technology, Tampere, Finland. Since 1997, he has been with the KTH Royal Institute of Technology, Stockholm, Sweden, where he is currently a Professor of Signal Processing and Head of the Department of Signal Processing. From 2000 to 2006, he held an adjunct position at the Swedish Defence Research Agency. He has been a Guest Professor at the Indian Institute of Science (IISc), Bangalore, India, and at the University of Gvle, Sweden. He is a co-founder of Movelo AB. Dr. Hndel has served as an associate editor for the IEEE TRANSACTIONS ON SIGNAL PROCESSING. He was a recipient of a Best Survey Paper Award by the IEEE Intelligent Transportation Systems Society in 2013. 